\title{
OPEN
}

\section{6 \\ The Value of Audiovisual Firms}

\begin{abstract}
Assessments of audiovisual firms are complicated by the specificity of the industry; the value of an audiovisual firm is related to the commercial exploitation of the audiovisual products for which the company holds the relevant rights. Traditional assessment methods, therefore, should be properly adapted to the specific nature of the business. The methodologies for evaluating audiovisual firms focus on the assessment of their exploitation rights, and their estimated values coincide with the value of their libraries of exploitation rights. This chapter proposes an evaluation model specifically dedicated to audiovisual firms, based on "cash flow method". The chapter highlights also some criticalities of the flow-based method and suggests some corrections to be applied to the standard methodology.
\end{abstract}

La Torre, Mario. The Economics of the Audiovisual Industry: Financing TV, Film and Web. Basingstoke: Palgrave Macmillan, 2014. DOI: 10.1057/9781137378477.0011. 


\subsection{Introduction}

The need to estimate the value of a firm is generally related to major financial transactions. In regard to the ordinary activities of firms, their estimated value can be requested for financing purposes by lenders who need to carry out creditworthiness analysis, determine the sustainability of the credit or demand specific guarantees. Firm evaluation is instead mandatory when dealing with extraordinary business operations - such as sales, mergers, transfer of share packages and extraordinary procedures such as bankruptcy.

Their theoretical value, determined through the use of specific methods, is generally adjusted through a number of subjective evaluations related to specific operational needs; in case of major transactions such as mergers and acquisitions, the estimated value constitutes the basis for negotiating the final price.

Assessments of audiovisual firms are complicated by the specificity of this sector; traditional assessment methods, therefore, should be properly adapted to the specific nature of the business. Evaluations of audiovisual firms are not common - given their reduced access to credit and rare use of structured finance - and, generally, are performed with methods based on financial flows.

With a view of encouraging a greater interaction between the financial system and the audiovisual industry, assessment of such firms represents an essential element, especially to facilitate their access to credit. For such purpose, this chapter intends to propose an evaluation model specifically dedicated to audiovisual firms, based on what the literature defines as "cash flow method". The analysis of the methodology is accompanied by the description of the evaluation process. Finally, the chapter highlights also some criticalities of the flow-based method, in light of the current and future market dynamics, and suggests some corrections to be applied to the standard methodology.

\subsection{Evaluating a firm: which methodology for the audiovisual industry?}

Literature and business practice have explored several business evaluation methods, with the aim of using the ones that best fit the single operations at issue. 
Regardless of the methodology for such evaluation, the value of an audiovisual firm is related to the commercial exploitation of the audiovisual products for which the company holds the relevant rights. Audiovisual firms, in fact, with a few exceptions, generally do not diversify their activities through investments in real or financial assets, nor are they characterized by high levels of traditional assets.

The methodologies for evaluating audiovisual firms, therefore, focus on the assessment of their exploitation rights. Hence, their estimated economic value must coincide with the value of their libraries of commercial exploitation rights.

Exploitation rights of audiovisual products are neither real nor financial assets, instead they fall under the category of the so-called "intangible assets". Here we distinguish between detectable and non-detectable assets; following a traditional definition, the former are related to specific financial assets (e.g., credits or deposits), while the latter relate to different factors, such as human capital, management quality, image or reputation of firms in their reference markets, and constitute what is commonly defined as "goodwill". Audiovisual exploitation rights fall under the category of the detectable intangible assets and are related to the exploitation of assets represented by the audiovisual products (Figure 6.1).
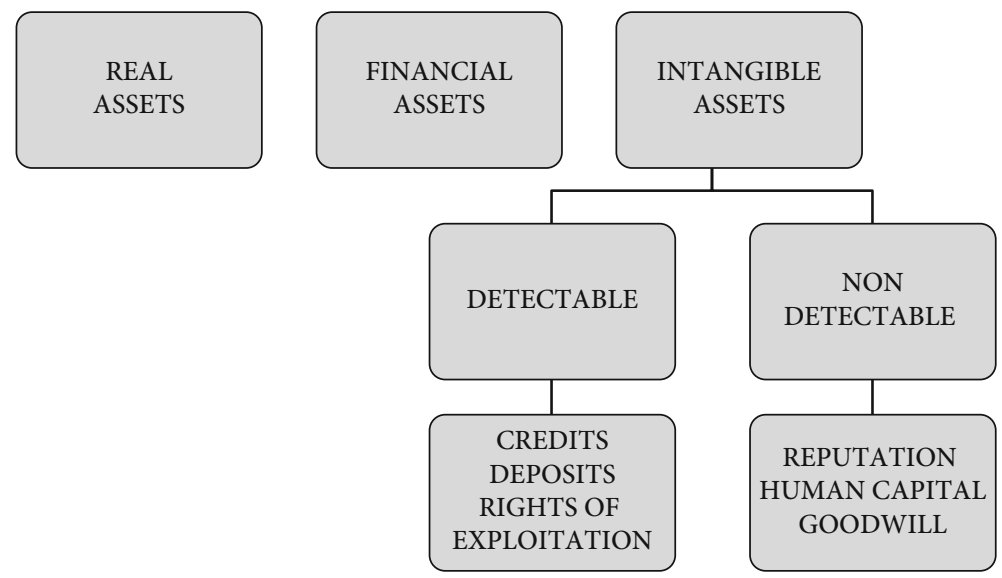

FIGURE 6.1 The nature of exploitation rights 
The estimate of the value of audiovisual firms, therefore, must follow methodologies used to assess intangible assets. Here, the literature identifies three options that are widely used in the common business practice: cash flow-based method, cost-based method and market-based method.

Cash flow-based models regard assets as investments and determine their value by discounting the cash flows generated. Generally speaking, therefore, according to these models, the formula indicating the value of the assets is expressed by the sum of the discounted cash flows (FL) and final asset values $(\mathrm{Wn})$

$$
\begin{aligned}
\mathrm{FL}= & (\mathrm{FLn} 1) /(1+\mathrm{i})+(\mathrm{FLn} 2) /(1+\mathrm{i})^{\circ} \ldots .+(\mathrm{FLn} \mathrm{n}) /(1+\mathrm{i})^{\circ} \\
& +\mathrm{Wn} /(1+\mathrm{i})^{\circ}
\end{aligned}
$$

Cash flow-based models, therefore, equal the asset value to the present cash flow value; cash flows are represented by the difference between revenues and costs (earnings method) or income and expenditures (financial method). In the case of libraries of exploitation rights related to audiovisual products, the inflows generated by the rights originate from the different forms of exploitation of such products on the market, while the outflows are given by various costs related to distribution or overhead pro rata costs of the single products.

However, within these models, we can further distinguish between different methods according to the nature of the flows and the capitalization regime. Specifically, we distinguish methods that take into account specific cash flows within a given period or, in case it is impossible to estimate the exact amount, methods based on normalized expected cash flows. As for the capitalization regime, we can differentiate between methods considering an unlimited number of flows and applying a perpetual capitalization regime, and methods that adopt a limited capitalized regime.

For libraries of audiovisual products, the preferred method of choice is the limited capitalized regime, generally to ten years, while to determine cash flows, the professionals in the sector adopt mostly a mixed method based on both specific estimates and standard values following specific parameters.

The methodology at issue, however, imposes two additional choices: one is related to the expected cash flow growth rate; the other concerns the discount rate.

In both cases, the market practice adopts a highly conservative approach that tends not to incorporate any cash flow growth rate and 
considers a specific risk premium for this sector when determining the discount rate.

Cost-based methods match the value of the assets with the cost attributable to them; besides real asset activities, they include also intangible assets, such as, for instance, trademarks, patents, rights, human capital and know-how. Here, we can distinguish three criteria: adjusted historical cost, replacement or reproduction cost and loss cost.

The historical cost method determines the value of the assets according to their production cost, aptly adjusted in order to incorporate a possible depreciation of the assets. The replacement or reproduction cost method calculates the value of an asset according to the cost needed to acquire a similar one; de facto, this method consists of discounting the investment necessary to produce an equivalent asset capitalized for a period of time needed to produce the asset itself.

The loss cost method determines the value of an asset by discounting the difference between the cash flows that the firm would receive if it had the asset and those resulting from the loss of the same. It is based on specific balance sheet values.

Finally, the market or empirical methods determine the value of the asset through a number of market indicators. Such methods revolve around some specific ratios related to firms operating in the same industry and of similar size (Price/Cash Flow, Price/Sales, Enterprise Value/ Earnings Before Interest, Taxes or Enterprise Value/Earnings Before Interest, Taxes, Depreciation/Amortization) with the extrapolation of an average value, which, compared with the company's balance sheet, will show the estimated value of the economic capital of the firm.

The choice of the most suitable methodology to represent the value of the assets examined must be carried out with the intent of maximizing the rationality, objectivity and neutrality of the evaluation. In other words, the value assigned to the assets must originate from a logical and consequential process (rationality), based on reliable and, if possible, verifiable data (provability) and without being affected by the distorting effects related to specific market dynamics or commercial relationships characterized by bargaining power circumscribed in space and time (neutrality).

The cash flow-based method appears to be the most suitable to determine the value of audiovisual libraries, as it is consistent with the above-mentioned criteria; it is also the most used by business practice. Cost-based methods do not suit audiovisual products, which, as prototypes, do not 
allow for a direct correlation between value and cost. Following marketbased methods, the assessment outcomes are obviously influenced by the choices made on the type of assets examined and the price selected. Moreover, the audiovisual industry has a very few benchmarks, mostly referred to the American market and, therefore, hardly compatible with national European and extra-EU markets.

On the other hand, cash flow-based methods allow taking into account the specific nature of audiovisual products as well as their libraries; they also facilitate compliance with the afore-mentioned criteria of rationality, objectivity and neutrality. The flexibility of this method allows combining specific estimates, where necessary and possible, with standardized values; the business practice has consolidated approaches that tend to limit the capitalization period, ignore any flows growth rate under the period examined and incorporate a specific risk premium for the sector. These factors ensure a prudential estimate and a customization of the evaluation.

\subsection{Evaluating a library of audiovisual products}

\section{Defining the potential markets for future inflows}

To determine the value of audiovisual libraries, the second step in the process involves the choice of the exploitation markets to consider (Figure 6.2).

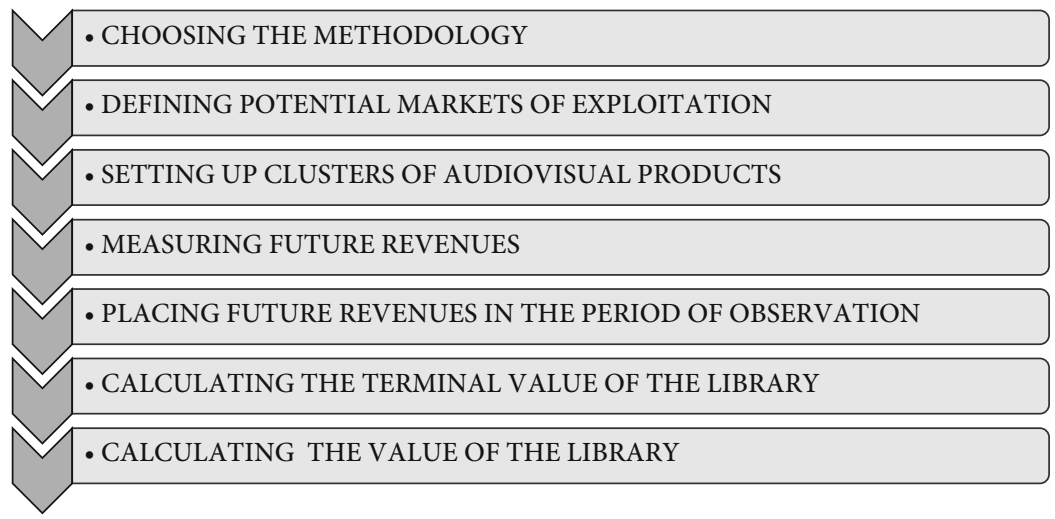

FIGURE 6.2 The steps to evaluate a library 
Given the specific nature of audiovisual products, the evaluation should take into account multiple markets; regarding cinematographic work, for instance, the most logical choice is to include all exploitation markets; television products instead should be assessed by selecting specific markets according to historical exploitation data, as per normal practice. In other cases, libraries may include exploitation rights related to a variety of different products; in such cases, the evaluation shall necessarily cover all exploitation markets, even if some of them will only involve some specific products.

A non-restrictive approach, therefore, should generally consider the following exploitation markets: Theatrical, Home Video (HV), Pay TV (Pay), Pay Per View (PPV), Video on Demand (VOD), Free TV (Free), New Media (NM), Accessories (Remake-Prequel-Sequel and Spin-off), Ancillary markets, foreign markets.

\section{Setting up clusters of audiovisual products}

Once the reference markets have been established, we need to decide which parameters to use to measure the future cash flows related to the single library products.

The next step consists of setting up clusters of audiovisual products within the libraries. The latter are divided into different categories, where audiovisual products are classified. The categories identify different kinds of potential to generate revenues; top categories are assigned higher revenue projections. Classes and the classification of audiovisual products are determined according to the historical records related to the performance of the titles examined; such records are surely available, for the exploitation rights of products that have already been produced. As libraries represent the most important assets of audiovisual firms, the evaluation cannot cover future production. If historical records are not available, it is only because the products, following their production phase, have not yet been released on the market. If this is the case, the placement of such products in one of the above categories will be based on future revenue projections, according to the artistic elements that characterize the products.

As for cinema works, title clusters are determined, first of all, according to the box office generated by the single movies: box office performance is considered a significant benchmark to calculate the commercial potential of a given product also in other exploitation markets. Alternatively, 
in order to avoid distortions related to different tickets prices over time, also in light of inflation rate, another option is to consider the number of viewers (tickets sold).

As for TV products, as well as all other products not expected to be released on the big screen, the reference economic parameter is given by the advertising revenues that can be associated to the products; alternatively, if the above data is absent, we can consider the share, as an audience measurement technique.

Products characterized by highly cultural content, whose performance obviously cannot be measured only according to strictly economic parameters, are generally included in a category of their own. This is the case of documentaries, for instance.

The clusters' thresholds are determined following data related to the single titles examined and, therefore, may vary according to the libraries. In general, it is necessary to identify clusters ensuring a limited dispersion of the revenue values observed. The number of quality grids may vary depending on the evaluations; in case of composite and comprehensive libraries, at least four clusters should be distinguished:

- Top class A, representing the firms' cutting-edge products, those with high commercial potential, in any case higher than the market average;

- Class A, including all titles that obtained revenues lower than Top Class A products, but still relevant; this class represents high-profile products with good commercial potential, in any case higher than the market average;

- Class $B$, representing titles that generated lower revenues than Class A products; this class includes the so-called "support" titles, those with discrete commercial potential, in line with the market average.

- Bottom class $C$ includes titles that generated minimal revenues and characterized by limited commercial potential; this class represents the lowest value in terms of expected revenue streams from marketing.

It must be pointed out that the placement of the titles in grids according to the parameters chosen may be subject to some corrections. For some titles, it might be necessary to carry out specific analysis in order to obtain a more accurate classification; sometimes, in fact, values in terms of exploitation rights may not be related to the box office figures or TV share. Such corrections may be due to artistic and commercial 
reasons related to different factors, such as the participation in festivals and awards received, the reputation of the directors or the cast - or their unexpected artistic and commercial success - the international success of the titles or the rediscovery of some genres that determines a sudden increase of their value.

\section{The methodology to estimate future revenues}

Once the clusters for the single library products have been identified, the next step is to choose the methods to determine estimated revenues related to the different forms of future commercial exploitation.

First of all, cash flows must be considered according to the percentages of exploitation rights held by the firms; some titles, in fact, may be co-produced or purchased only after production; in such cases, a firm can only hold a percentage of their exploitation rights or rights only for some specific markets and only for a limited period. Assessors, therefore, should carefully verify the ownership of the exploitation rights for each single product, their percentages, the holding period and exploitation markets.

Cash flow assessment must highlight a picture of the flow of funds actually available. Generally, cash flow projections are considered gross of production and acquisition costs, as well as overhead costs - for the latter have already occurred at the assessment date - and net of distribution costs and commissions - if not incurred. Regarding cinema works, we therefore need to subtract the revenues due to distributors and exhibitors from the expected cash flows generated by their theatrical release.

In each exploitation market, then, we should divide between first sales and subsequent sales (mainly second and third sales) of the exploitation rights. To determine the value of the former, the benchmark could be represented by the amounts indicated in the contracts entered by the firms, or by market data, if available. Any time firms have in place contracts on the titles examined, the reference revenues are those expressed in the contracts: of course, here we should only consider revenue flows not yet collected at the time of the evaluation.

In some markets, such as Pay TV, there are official parameters to determine the value of the rights: it is not uncommon that Pay TV broadcasters adopt "escalators" to calculate the standardized purchase price of audiovisual rights; for films released at cinemas, the escalators' benchmark is represented by the box office results. Be advised that the prices indicated in the escalators, though constituting a significant 
reference basis, may significantly differ from the actual negotiation prices, due both to the number of runs planned and the exploitation period planned, and also to package sales, where the single titles are usually sold at flat rates, often a much more convenient system than selling them separately. It is up to the discretion of the assessors to evaluate the capacity of the single titles to access the exploitation markets considered; especially for the lower library clusters, it may be savvy to adopt a prudential approach and rule out some exploitation forms. When titles express no significant independent potential, but could be included in package sales, it is possible to indicate a standardized value for the whole category for some markets. Here too the assessors' discretion plays a relevant role.

As for second sales on different exploitation channels, in general, the most widespread methodology determines the revenues by reducing first sale revenues by a given percentage. Usually, low percentage reductions are applied to top categories, then they gradually increase for the bottom quality grids. These percentages of reduction will be even higher in case of further sales following the second. In this case too, it may be appropriate to rule out revenues for some specific titles or categories; again, it is in the assessors' discretion to establish the commercial potential of the products over time.

\section{The distribution of future revenues}

Expected future revenues must be considered over a period of time; it is, therefore, necessary to establish the evaluation reference period. As anticipated, generally, the audiovisual industry relies on a ten-year period; according to standard contracts, we can assume that titles can be exploited up to the third sale. It goes without saying that each title has a different commercial potential in different markets and, therefore, not all titles and categories can be evaluated throughout a complete sales cycle.

The estimated cash flows have to be distributed over the evaluation reference period following a prudential approach. In particular:

- for the temporal placement of the first sales, we need to consider a physiological time frame for relatively new titles, while it is appropriate not to include in the evaluation future revenues related to the oldest library titles that, at the time of assessment, have not yet accessed some exploitation markets; 
- for the temporal placement of the second sales, it is appropriate to consider a break following the first sale, assuming that firms are not able to immediately renegotiate their rights; and

- as for the third sales, a prudential approach would suggest to place them at the end of the period, regarding them as residual opportunities to generate less significant revenues for evaluation purposes.

\section{The terminal value of the library}

In addition to the expected revenues, the assessment considers also a "terminal value" of the whole library at the end of the exploitation period. Such expectation is justified by the firms' going-concern, which is taken as a fundamental assumption in the methodology of choice. This value is included on the assumption that firms will continue to operate also following the period considered by the evaluation.

Of course, the terminal value can only be referred to products for which the firms hold perpetual ownership/co-ownership rights. Following a prudential approach, this method does not consider the exploitation of temporary license rights over periods following the evaluation reference period.

The terminal value of libraries at the end of the evaluation reference period is generally calculated by applying a percentage reduction only to the value of the titles owned; the terminal value will be much lower as far away as is the last year of assessment in question, and the more intense and repeated the cycle of exploitation adopted for the estimate.

\section{The value of the library}

Once we have identified the future cash flows originating from the libraries, we must proceed to discounting according to the observation period chosen. The discount rate is established by considering, first of all, a benchmark rate for the risk-free rate; generally, the most popular benchmarks are the interest rates paid by government bonds with duration equal to, or close to, that of the evaluation period. A premium for general enterprise risk is added to the risk-free rate. Following a prudential approach, given the intangible nature of the assets at issue and the volatility expressed by the audiovisual market - a further specific risk premium can be added. 
The risk-free rate and the risk premiums are determined by the assessors on an individual basis and vary according to the economic cycle, as well as the specific economic cycle of the industry.

The sum of future revenue flows and the library terminal value, discounted at the rate established, shall determine the library value. For economic and strategic evaluation purposes, this value can be broken down in relation to both the clusters and the exploitation markets considered (Tables 6.1 and 6.2). In the first case, the assessment allows identifying the quality composition of the portfolio of rights held by

TABLE 6.1 Value of the library by class of products

\begin{tabular}{ll}
\hline CLUSTERS & NET PRESENT VALUE \\
\hline TOP CLASS A & \\
TOTAL TOP CLASS A & \\
CLASS A & \\
CLASS B & \\
CLASS C & \\
DOCUMENTARY \\
TOTAL VALUE FUTURE REVENUES \\
TERMINAL LIBRARY VALUE \\
TOTAL LIBRARY VALUE \\
\hline
\end{tabular}

TABLE 6.2 Value of the library by exploitation markets

\begin{tabular}{ll}
\hline MARKETS OF EXPLOITATION & NET PRESENT VALUE \\
\hline THEATRICAL & \\
PAY & \\
PPV & \\
VOD & \\
HV & \\
FREE & \\
NEW MEDIA & \\
ANCILLARY & \\
FOREIGN MARKETS & \\
REMAKE, PRE-SEQUEL E SPIN-OFF & \\
DOCUMENTARY & \\
TOTAL VALUE REVENUES PER MARKETS & \\
TERMINAL LIBRARY VALUE \\
TOTAL LIBRARY VALUE
\end{tabular}


firms; further examinations may help determine the technical, artistic and economic characteristics of the titles included in the different classes.

The classification based on exploitation markets offers indications on the business capabilities of the firms, as well as the commercial potential of the products in their portfolios.

In general, besides the quantitative data summarized by the library value, the evaluation can provide useful qualitative indications for the strategic repositioning of products and firms alike.

\section{Some critical issues}

The evaluation methodology described aims to determine the library value of firms; as a portfolio of exploitation rights often represent the main asset - if not the sole asset - of a given audiovisual firm, library evaluation can be considered a proxy of the audiovisual firm assessment as a whole.

However, the recent market dynamics impose a critical rethinking of the methodologies currently used to estimate the value of audiovisual libraries in the business. These methods, in fact, have been used too mechanically. The division of the titles in clusters inevitably results in a standardized evaluation of titles belonging to the same cluster. This solution, which is useful in case of libraries composed of a variety of titles, clashes with the very "nature" of the "exploitation rights" as intangible assets. The assets underlying exploitation rights are audiovisual products, which are prototypes by definition; in light of this, it is reasonable to assume that each title may have its own commercial history that cannot be assessed through standardized parameters.

The choice of the evaluation period itself, which tends to consider medium- and long-term opportunities for exploitation, while being consistent with the exploitation cycle of audiovisual products, clashes with market volatility. The economic crisis has clearly showed that the value of assets - whether real, financial or intangible assets - is subject to economic cycles and characterized by significant degrees of volatility. Although audiovisual is one of the most uncorrelated businesses from the traditional sectors, some of its internal financial dynamics make it vulnerable to economic cycles. In particular, all those products for which the biggest source of revenues is constituted by their exploitation on the TV market are heavily exposed to the performance 
of advertising, and through this, to the economic cycles of the single countries. Consequently, any time future revenues are estimated on overly extended periods of time, the risk is to lose the significance of the estimate and obtain misleading indications.

In this perspective, the very same definition of discount rate might be denied in the short term. The interest rate trends recorded in different countries and main economic areas in recent years call for increasingly prudential choices.

Audiovisual libraries' and firms' evaluations are needed to facilitate a convergence process between the financial markets and the audiovisual industry. It is, therefore, necessary to push for a library-oriented culture and proper evaluation methods. Markets, though, should promote tailormade evaluation methods to a greater degree; audiovisual products must be treated as prototype assets indeed, whose commercial potential is related to the efficiency of the distribution chain and the emotional response from the public. A greater attention on the single titles, precise reconstruction of historical data and a more flexible articulation of the clusters may all help achieve tangible benefits for evaluation purposes.

On the other hand, it must be pointed out that medium- and longterm evaluation perspectives do not fit the nature of the industry and its interactions with the economic system. It is, therefore, necessary to adjust the evaluation to the individual cases and adopt an increasingly prudential approach as much as the observation period is extended, along with more frequent updates of the estimate.

(c) (i) Except where otherwise noted, this work is licensed under a Creative Commons Attribution 3.0 Unported License. To view a copy of this license, visit http://creativecommons.org/licenses/by/3.0/ 